\title{
Apigenin as a promising myocyte protectant against damage and degradation
}

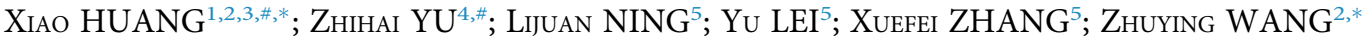 \\ ${ }^{1}$ Institute of Cultural and Technological Industry Innovation of Tongren, Tongren, 554300, China \\ ${ }^{2}$ School of Sports and Physical Education, Nanjing Normal University, Nanjing, 210046, China \\ ${ }^{3}$ School of Physical Education, Guangxi University of Science and Technology, Liuzhou, 545006, China \\ ${ }^{4}$ Department of Urology Surgery, Chongqing University Three Gorges Hospital, Chongqing, 404000, China \\ ${ }^{5}$ School of Sports and Health Science, Tongren University, Tongren, 554300, China
}

Key words: Apigenin, Myocyte, Protectant, Pharmacological activity

\begin{abstract}
Myocytes power the movement of all organs in the body. Damage to and degradation of myocytes causes hypokinesia and muscle-related degenerative diseases. Apigenin, a kind of flavone, is being used to treat many disorders. It exerts a host of different pharmacological activities, such as anti-inflammatory, anti-mutagenic, cardioprotective, and antioxidant effects. Accordingly, apigenin is considered a promising candidate for myocyte protection. In this review, we introduced the characteristics of apigenin. The means of apigenin protection of myocytes as well as the mechanism were summarized and discussed. The protective effects can be classified into proliferation-promoting, anti-inflammatory, atrophy-preventing, metabolism-increasing, and antioxidative effects. Additionally, we provided some outlook on the valuable applications of apigenin in sports medicine, which eagerly require further fundamental research.
\end{abstract}

\section{Introduction}

Myocytes can contract and relax, allowing them to a crucial role in body movement. Thus, they are the power source for movement. However, improper exercise causes exercise fatigue originally resulting from energy burn-out and myocyte hypoxia, producing numerous free radicals (Verhavert et al., 2020; Zhou and jiang, 2019). Oxidative imbalance leads to some serious consequences, including decreased $\mathrm{pH}$, inflammatory responses, and hypoimmunity (Jayakumar et al., 2020; Li et al., 2020; Baldelli et al., 2019; Mulkey et al., 2003). This imbalance can accelerate necrosis and the apoptosis of myocytes, reducing exercise performance (Narasimhan and Rajasekaran, 2016). In addition, aging and obesity can also induce myocyte atrophy (Yatsenko et al., 2020; Lee et al., 2016). Therefore, protecting myocytes against damage and degradation becomes very important for preventing hypokinesia and muscle-related degenerative diseases.

\footnotetext{
*Address correspondence to: Xiao Huang, humphrey8531@hotmail.com; Zhuying Wang, wangzhuying@njnu.edu.cn

\#These two authors contribute equally to this work

Received: 01 January 2021; Accepted: 23 February 2021
}

It is widely believed that nutrient supplementation is an effective way to keep muscles healthy (Ganapathy and Nieves, 2020). Among the various sports supplements, herbs and their extracts have attracted more attention due to their high efficiency and low toxicity (Zhang et al., 2014). Polyphenols can reduce oxidative damage and promote myocyte survival (Carresi et al., 2016). Astragalus polysaccharide can inhibit the autophagy and apoptosis of $\mathrm{C} 2 \mathrm{C} 12$ myoblasts by regulating apoptosis-related protein (B-cell lymphoma-2 (Bcl-2), cytochrome $\mathrm{C}$ (cyto-C), and cysteine protease 3 (caspase-3)) (Yin et al., 2015). Astragalus polysaccharide also prevents muscle cell atrophy by activating the ubiquitin-proteasome pathway (Geng et al., 2017). Gentianella acuta can inhibit the nuclear factor $\kappa \mathrm{B} /$ cyclooxygenase-2 (NF- $\kappa \mathrm{B} / \mathrm{COX}-2)$ signaling pathway to down-regulate inflammatory factors, preventing myocardial ischemia/reperfusion injury (Ding, 2016). Recently, apigenin (API) has been recognized as a bioactive compound that possesses a host of different pharmacological activities, such as anti-inflammatory, anti-mutagenic, cardioprotective, and antioxidant effects (Kashyap et al., 2018). API was reported to relieve muscle atrophy by inhibiting oxidative stress apoptosis in the skeletal muscle of mice (Wang et al., 2020). API can also reduce eye fatigue by relaxing ciliary muscles 
(Kim et al., 2018). Accordingly, API is considered a promising candidate for myocytes protection. Several research teams and our group have focused on this issue. Herein, we summarized the protective effects and mechanism of API on myocytes. The information in this review can provide a theoretical basis for API as a promising myocyte protectant against damage and degradation.

\section{Characteristics of API}

API is a kind of flavonoid and exists in a wide variety of vegetables, fruits, beans, and teas. The API content is relatively high in celery $(2154 \mathrm{mg} / \mathrm{kg}$ dry weight), guava (579 mg/kg dry weight), wolfberry leaves (547 mg/kg dry weight), bilimbi fruit (458 $\mathrm{mg} / \mathrm{kg}$ dry weight), pepper (272 mg/kg dry weight), kumquat (219 mg/kg dry weight), garlic $(217 \mathrm{mg} / \mathrm{kg}$ dry weight), Chinese cabbage $(187 \mathrm{mg} / \mathrm{kg}$ dry weight), bell French peas (176 mg/kg dry weight), snake gourd (42 mg/kg dry weight), daun turi (39 mg/kg dry weight), and kadok (34 mg/kg dry weight) (Nabavi et al., 2018; Yang et al., 2008; Miean and Mohamed, 2001). API is chemically known as 4',5,7-trihydroxyflavone with the molecular formula $\mathrm{C}_{15} \mathrm{H}_{10} \mathrm{O}_{5}$ and a molecular weight of 270.24. The basic carbon skeleton of API has a flavan nucleus. Its structure consists of 15 carbons arranged in two aromatic rings connected by a 3 -carbon bridge, forming a diphenyl propane structure. Three hydroxyl groups are present at positions 5 and 7 on the chromone and 4', respectively. It also has an oxo group on the chromone at position 4 (Fig. 1) (Hassanpour et al., 2020; Ali et al., 2017).

API has acquired importance over the past few years as a salutary and health-promoting agent because of its low intrinsic toxicity. According to in vitro, in vivo, and clinical trial studies, API has been used to treat autoimmune diseases because of its anti-inflammatory effect (Kairi et al., 2018), nervous system diseases by virtue of its antioxidant function (Ginwala et al., 2016), cancer, from its ability to regulate the cell cycle (Kashyap et al., 2018), and other diseases due to its pharmacological properties (Ali et al., 2017).

\section{Protective effect of API on myocytes}

\section{Promoting proliferation}

The phosphatidylinositol 3-kinase/serine-threonine protein kinase/mammalian target of the rapamycin (PI3K/AKT/mTOR) signaling pathway plays an important role in regulating the cell cycle. AKT is the downstream target protein of PI3K. When AKT is activated, cells can escape from apoptosis, and proliferation is promoted (Rychahuo et al., 2005). In C2C12 cells, AKT can be activated by API, and the<smiles>O=c1cc(-c2ccc(O)cc2)oc2cc(O)cc(O)c12</smiles>

FIGURE 1. Chemical structure of API. expression of phosphorylated AKT (pAKTSer473) increases. Then, pAKTSer473 phosphorylates glycogen synthase kinase$3 \beta$ (GSK3 $\beta$ ), which mediates the degradation of cyclinD1 protein to promote cell proliferation. And the cellular viability of $\mathrm{C} 2 \mathrm{C} 12$ increased about $10 \%$ compared to the control group. (Kulabas et al., 2018). Besides, AKT can phosphorylate cell cycle inhibitors ( $221^{\mathrm{WAF} 1}$ and $\mathrm{p} 27^{\mathrm{Kip} 1}$ ) to inhibit cyclinD1/cyclin-dependent kinases 4 (CDK4), promoting cell cycling from the G1 phase to the $\mathrm{S}$ phase (Cheung and Testa, 2013; Hua et al., 2008).

Mitochondria are organelles in cells that supply energy for cell proliferation. The mitochondrial biogenesis and mitochondrial function of skeletal muscle cells were shown to increase after API treatment. Increased mitochondrial size and the mtDNA (peroxisome proliferator-activated receptor- $\gamma$ coactivator-1a (PGCla), mitochondrial transcription factor (mtTFAM). and cytochrome c (CyCs)) contents have been observed in API-treated mouse skeletal muscle cells. Therefore, skeletal muscle fiber size was significantly increased, and there was a tendency toward an increase in the skeletal muscle mass (Choi et al., 2017). These results suggest that API can ameliorate mitochondrial function and promote myocyte proliferation.

\section{Anti-inflammatory effects}

It is well known that many flavonoids selectively or nonselectively inhibit cyclooxygenase (COX), lipoxygenase (LOX), phospholipase (PLA), and nitric oxide synthase (NOS), which are the major contributors to inflammation. API has been found to suppress lipopolysaccharide (LPS)induced nitric oxide (NO) production and COX-2 expression (Gutierrez-Venegas and Gonzalez-Rosas, 2017). In an acute lung injury mouse model, API lowered the production of some inflammatory cytokines (interleukin-6 (IL-6), interleukin-1 $\beta$ (IL-1 $\beta$ ) and tumor necrosis factor $\alpha$ $(\mathrm{TNF}-\alpha)$ ) via inhibiting the COX-2 and NF- $\kappa \mathrm{B}$ activation pathways (Wang et al., 2014). Further, studies also reported that API exerted anti-chronic inflammatory effects. API was capable of normalizing the expression of many colonic inflammatory markers in a bowel disease model (Mascaraque et al., 2015). These studies demonstrate the anti-acute and chronic inflammatory effects of API.

Choi et al. (2017) investigated the effects of API on inflammatory cytokines such as monocyte chemoattractant protein 1 (MCP-1), IL-1 $\beta$, TNF- $\alpha$, and IL-6 in the skeletal muscle of obese mice. After API treatment, the mRNA expression of those inflammatory cytokines decreased significantly. TNF- $\alpha$ and IL- 6 levels were also lower in the serum of the API-treated mice. API probably inhibited the NF- $\kappa B$ activation pathways in the myocytes of the obese mice. In view of the anti-acute and chronic inflammatory effects of API, it can be inferred that both acute inflammation induced by exhaustive exercise and chronic inflammation induced by chronic diseases can be normalized by API. In this way, myocytes can be protected from inflammatory injury.

\section{Preventing atrophy}

The pathological characteristics of muscle atrophy show the progressive loss of muscle mass and strength. It is a complex process, and its exact causes have not been identified. Some studies reported that skeletal muscle was 
atrophied as a result of inflammatory conditions such as sepsis (Liu et al., 2019). Recent research has suggested that skeletal muscle atrophy is potentially induced by some atrophic stimuli (Rosa-Caldwell and Greene, 2019; Rienzo et al., 2019; Li et al., 2017), which disturb the balance between the synthesis and breakdown of myofibrillar proteins. Acting on specific targets can regulate signal transduction pathways to promote the synthesis or degradation of muscle proteins to control muscle hypertrophy or atrophy (Pipis et al., 2019).

The results of studies by Shiota et al. (2015) showed that API could significantly inhibit LPS-induced c-Jun N-terminal kinase (JNK) phosphorylation in $\mathrm{C} 2 \mathrm{C} 12$ myotubes. In this manner, LPS-mediated muscle atrophy can be prevented by API via the downregulation of muscle atrophy F-box (MAFbx/atrogin-1) expression. API also regulated the expression of other atrophic genes, such as myosin heavy chain (MyHC) IIa and muscle-specific ring finger protein 1 (MuRF1), to prevent muscle atrophy. Moreover, API can increase the nuclei ratio of $\mathrm{C} 2 \mathrm{C} 12$ cell nucleus compared to the palmitic acid-treated cells, which is a similar environment to the obese state, by downregulating the expression of MuRF1 (Choi et al., 2017). Therefore, API has great potential use for preventing muscle atrophy.

\section{Increasing metabolism}

Skeletal muscle is the most important peripheral tissue contributing to the uptake of glucose into cells, a process underlying insulin control. The glucose uptake of myocytes, as well as glucose utilization, is reduced in people with insulin resistance. Consequently, myocytes suffer from functional degeneration, and type 2 diabetes mellitus is more likely to develop (Meng et al., 2020). Insulin-induced glucose uptake initially takes place through translocation of glucose transport-4 (GLUT4) on the cell membrane. And in insulin resistance, GLUT-4 is down-regulated, resulting in decreased insulin-stimulated glucose uptake and metabolism. API, as the effective constituent, significantly increased GLUT4 expression in muscle cells both in vitro and in vivo (Kulabas et al., 2018; Li et al., 2007). This is because API activated the upstream protein AKT (Tan et al., 2020).

Additionally, API also increased enzyme lipoprotein lipase (LPL) and peroxisome proliferator-activated receptor $\gamma$ (PPAR $\gamma)$ levels in an insulin-resistant $\mathrm{C} 2 \mathrm{C} 12$ cell model (Kulabas et al., 2018). LPL is a key enzyme in lipid metabolism. Low LPL activity may induce abnormal lipid metabolism (Hao et al., 2016). And PPAR $\gamma$ plays an important role in lipid-mediated insulin resistance in muscle and hepatic cells (Kim et al., 2020). Those results indicated that API could participate in normalizing lipid metabolism. Another metabolism-related key protein, adenosine 5'-monophosphate (AMP)-activated protein kinase (AMPK), was also activated in obese mice by API (Choi et al., 2017). AMPK is effective in balancing glucose metabolism (Trinchese et al., 2020) and enhancing mitochondrial function (Sun et al., 2020).

\section{Antioxidant function}

The three hydroxyl groups at positions 5, 7, and 4', and the C-C double bond between Positions 2 and 3 in the chemical structure of API can react with free radicals. The two hydroxyl groups at positions 5 and 7 can chelate metal ions to inhibit the production of free radicals. Those characteristics confer API with antioxidant activity. API not only scavenges free radicals directly but also enhances the antioxidant ability of cells by regulating heme oxygenase (HO-1), glutamate-cysteine ligase, catalytic subunit (GCLC) and glutamate-cysteine ligase, modifier subunit (GCLM) gene transcription via the extracellular regulated protein kinases 2 (ERK2)/nuclear factor E2-related factor 2 (Nrf2)/antioxidant response element (ARE) signaling pathways (Hassanpour and Niknam, 2020; Huang et al., 2013). Many studies have found that API helps in preventing oxidative stress injury by increasing the expression of antioxidant enzymes both at the mRNA and protein levels in several cells (Ogura et al., 2020; Zhang et al., 2019; Xu et al., 2016). However, to the best of our knowledge, there is no published study focusing on the direct antioxidant effects of API on myocytes. Our group found that API significantly increased the viability of skeletal muscle cells under oxidative stress induced by tert-butyl hydroperoxide. It is probable that API activates the Nrf2 and mitogen-activated protein kinase (MAPK) signaling pathways (data not published). Therefore, it can be inferred that API has effective antioxidant effects, protecting myocytes against oxidative stress injury.

\section{Conclusion and outlook}

Myocytes are the power source for the movement of all organs of the body. Myocyte damage and degradation cause hypokinesia and muscle-related degenerative diseases. API, a kind of flavone, has been used as a salutary and health-promoting agent because of its low intrinsic toxicity. The evidence gathered in this review indicates that API possesses hopeful pharmacological activity as an agent protecting myocytes against damage and degradation. API protects myocytes by promoting proliferation, exerting anti-inflammatory and antioxidant effects, preventing atrophy, increasing metabolism, and activating the respective signaling pathways (Fig. 2).

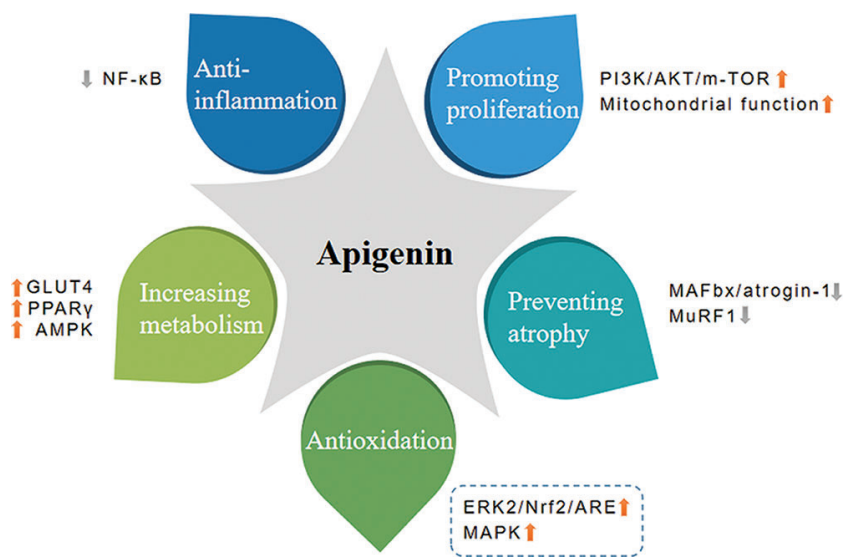

FIGURE 2. Protective effect on myocytes and its mechanism (promoting proliferation: up-regulate $\mathrm{PI} 3 \mathrm{~K} / \mathrm{AKT} / \mathrm{mTOR}$ pathway (Kulabas et al., 2018) and enhance mitochondrial function (Choi et al., 2017); anti-inflammation: down-regulate NF- $\kappa B$ pathway (Choi et al., 2017); preventing atrophy: down-regulate MAFbx/atrogin-1 (Shitota et al., 2015) and MuRF1 (Choi et al., 2017) expression; increasing metabolism: increase GLUT4 express (Tan et al., 2020; Kulabas et al., 2018; Li et al., 2007), PPAR $\gamma$ level (Kulabas et al., 2018) and activate AMPK (Trinchese et al., 2020; Sun et al., 2020) and antioxidation: probably up-regulate ERK2/Nrf2/ARE and MAPK pathways) of API. 
However, studies on the protective effect of API on myocytes are still scarce. And all of the research has focused on the obesity model. In fact, API also performs effectively in acute conditions. Therefore, its protective effect on myocytes in exercise models needs to be investigated. The results of such studies would make valuable contributions to sports medicine.

The remarkable effect of API on cardioprotection also needs to be noted (Zhang et al., 2015). API can reduce myocardial ischemia/reperfusion injury and attenuate anoxia/reoxygenation-induced myocardium injury (Quan et al., 2020; Feng et al., 2018; Chen et al., 2016; Hu et al., 2015; Yang et al., 2015). API can depress contractions in arterial smooth muscle cells induced by various vasoconstrictors (Jing et al., 2019). These vascular pharmacological activities of API would play an equally important role in whole-body exercise in the field of sports medicine.

Overall, API holds great potential in protecting myocytes against damage and degradation to prevent hypokinesia and muscle-related degenerative diseases. Therefore, fundamental research into API is warranted.

Availability of Data and Materials: Not Applicable.

Author Contribution: The authors confirm contribution to the paper as follows: project conception: Xiao Huang and Zhuying Wang; paper preparation: Xiao Huang and Zhihai $\mathrm{Yu}$; references collection and analysis: Lijuan Ning, Yu Lei, and Xuefei Zhang. All authors reviewed and approved the final version of the manuscript.

Ethics Approval: Not Applicable.

Funding Statement: This work is supported by the China Postdoctoral Science Foundation (Grant No. 2019M653494); Special Field Projects of Guizhou Education Committee (Grant No. KY2019072); and High-level Innovative Talents in Guizhou Province (Grant No. 2018-2016-023).

Conflicts of Interest: The authors declare that they have no conflicts of interest to report regarding the present study.

\section{References}

Ali F, Rahul, Naz F, Jyoti S, Siddique YH (2016). Health functionality of apigenin: a review. International Journal of Food Properties 20: 1197-1238. DOI 10.1080/10942912.2016.1207188.

Baldelli S, Ciccarone F, Limongi D, Checconi P, Palamara AT, Ciriolo MR (2019). Glutathione and nitric oxide: key team players in use and disuse of skeletal muscle. Nutrients 11: 2318. DOI 10.3390/nu11102318.

Carresi C, Musolino V, Gliozzi M, Giancotta C, Scarano F, Oppedisano F, Paone S, Palma E, Muscoli C, Mollace V (2016). Bergamot polyphenols protect against doxorubicininduced cardiomyopathy reducing ROS production and promoting myocyte survival. European Journal of Heart Failure 18: 134.

Chen CJ, He H, Luo Y, Zhou M, Yin D, He M (2016). Involvement of Bcl-2 signal pathway in the protective effects of apigenin on anoxia/reoxygenation-induced myocardium injury. Journal of Cardiovascular Pharmacology 67: 152-163. DOI 10.1097/ FJC.0000000000000331.
Cheung M, Testa JR (2013). Diverse mechanisms of AKT pathway activation in human malignancy. Current Cancer Drug Targets 13: 234-244. DOI 10.2174/1568009611313030002.

Choi WH, Son HJ, Jang YJ, Ahn J, Jung CH, Ha TY (2017). Apigenin ameliorates the obesity-induced skeletal muscle atrophy by attenuating mitochondrial dysfunction in the muscle of obese mice. Molecular Nutrition \& Food Research 61: 1700218. DOI 10.1002/mnfr.201700218.

Ding Y (2016). The study of applying gentianella acuta hulten for antiinflammatory mechanism on MIRI rats (Ph.D. Dissertation). Heilongiiang University of Chinese Medicine Harbin, Heilongiiang, China.

Feng YD, Lu YY, Liu D, Zhang W, Liu JT, Tang HF, Zhu YR (2018). Apigenin-7-O-beta-D-(-6"-p-coumaroyl)-glucopyranoside pretreatment attenuates myocardial ischemia/reperfusion injury via activating AMPK signaling. Life Sciences 203: 246-254. DOI 10.1016/j.lfs.2018.04.048.

Ganapathy A, Nieves JW (2020). Nutrition and sarcopenia-what do we know? Nutrients 12: 1755. DOI 10.3390/nu12061755.

Geng Z, Wei L, Zhang C, Yan X (2017). Astragalus polysaccharide, a component of traditional Chinese medicine, inhibits muscle cell atrophy (cachexia) in an in vivo and in vitro rat model of chronic renal failure by activating the ubiquitinproteasome pathway. Experimental and Therapeutic Medicine 14: 91-96. DOI 10.3892/etm.2017.4492.

Ginwala R, McTish E, Raman C, Singh N, Nagarkatti M, Nagarkatti P, Sagar D, Jain P, Khan ZK (2016). Apigenin, a natural flavonoid, attenuates EAE severity through the modulation of dendritic cell and other immune cell functions. Journal of Neuroimmune Pharmacology 11: 36-47. DOI 10.1007/ s11481-015-9617-x.

Gutierrez-Venegas G, Gonzalez-Rosas Z (2017). Apigenin reduce lipoteichoic acid-induced inflammatory response in rat cardiomyoblast cells. Archives of Pharmacal Research 40: 240-249. DOI 10.1007/s12272-016-0756-2.

Hao ZM, Ye YF, Zhang YK, Yang SF, Ye XL (2016). Lipoprotein lipase and lipid profiles in plasma and placenta from normal pregnancies compared with patients with intrahepatic cholestasis of pregnancy. European Journal of Obstetrics \& Gynecology and Reproductive Biology 203: 279-285. DOI 10.1016/j.ejogrb.2016.06.019.

Hassanpour H, Nikman V (2020). Establishment and assessment of cell suspension cultures of Matricaria chamomilla as a possible source of apigenin under static magnetic field. Plant Cell, Tissue and Organ Culture 142: 583-593. DOI 10.1007/s11240-020-01885-4.

Hassanpour H, Nikman V, Ahmadi-Sakha S, Haddadi B (2020). Antioxidant activity and flavonoid content of Matricaria chamomilla extracts from different populations of Iran. Journal of Botanical Research 2: 8-13. DOI 10.30564/jrb. v2i2.1909.

Hu J, Li ZL, Xu LT, Sun AJ, Fu XY, Zhang L, Jing LL, Lu AD, Dong YF, Jia ZP (2015). Protective effect of apigenin on ischemia/ reperfusion injury of the isolated rat heart. Cardiovascular Toxicology 15: 241-249. DOI 10.1007/s12012-014-9290-y.

Hua K, Feng W, Cao Q, Zhou X, Lu X, Feng Y (2008). Estrogen and progestin regulate metastasis through the PI3K/AKT pathway in human ovarian cancer. International Journal of Oncology 33: 959-967.

Huang CS, Lii CK, Lin AH, Yeh YW, Yao HT, Li CC, Wang TS, Chen HW (2013). Protection by chrysin, apigenin, and luteolin against oxidative stress is mediated by the Nrf2-dependent up-regulation of heme oxygenase 1 and glutamate cysteine 
ligase in rat primary hepatocytes. Archives of Toxicology 87: 167-178. DOI 10.1007/s00204-012-0913-4.

Jayakumar T, Huang HC, Hsia CW, Fong TH, Khamrang T, Velusamy M, Manubolu M, Sheu JR, Hsia CH (2020). Ruthenium derivatives attenuate LPS-induced inflammatory responses and liver injury via suppressing NF- $\kappa \mathrm{B}$ signaling and free radical production. Bioorganic Chemistry 96: 103639. DOI 10.1016/j.bioorg.2020.103639.

Jing Y, Chen R, Dong M, Liu Y, Hou X, Guo P, Li W, Lv J, Zhang M (2019). Apigenin relaxes rat intrarenal arteries, depresses $\mathrm{Ca}^{2+}$-activated $\mathrm{Cl}^{-}$currents and augments voltagedependent $\mathrm{K}^{+}$currents of the arterial smooth muscle cells. Biomedicine \& Pharmacotherapy 115: 108926. DOI 10.1016/j.biopha.2019.108926.

Kairi N, Rahmati M, Ahmadi I, Eskandari N (2018). The significant impact of apigenin on different aspects of autoimmune disease. Inflammopharmacology 26: 1359-1373. DOI 10.1007/ s10787-018-0531-8.

Kashyap D, Sharma A, Tuli HS, Sak K, Kumar V, Buttar HS, Setzer WN, Sethi G (2018). Apigenin: A natural bioactive flavone-type molecule with promising therapeutic function. Journal of Functional Foods 48: 457-471. DOI 10.1016/j.jff.2018.07.037.

Kim DH, Kim BM, Chung KW, Choi YJ, Yu BP, Chung HY (2020). Interaction between $\mathrm{CHOP}$ and FoxO6 promotes hepatic lipid accumulation. Liver International 40: 2706-2718. DOI 10.1111/liv.14594.

Kim J, Kang H, Choi H, Jo A, Oh DR, Kim Y, Im S, Lee SG, Jeong KI, Ryu GC, Choi C (2018). Aqueous extract of Perilla frutescens var. acuta relaxes the ciliary smooth muscle by increasing NO/cGMP content in vitro and in vivo. Molecules 23: 1777. DOI 10.3390/molecules23071777.

Kulabas SS, Ipek H, Tufekci AR, Arslan S, Demirtas I, Ekren R, Sezerman U, Tumer TB (2018). Ameliorative potential of Lavandula stoechas in metabolic syndrome via multitarget interactions. Journal of Ethnopharmacology 223: 88-98. DOI 10.1016/j.jep.2018.04.043.

Lee DE, Brown JL, Rosa ME, Brown LA, Perry RA, Wiggs MP, Nilsson MI, Crouse SF, Fluckey JD, Washington TA, Greene NP (2016). microRNA-16 is downregulated during insulin resistance and controls skeletal muscle protein accretion. Journal of Cellular Biochemistry 117: 1775-1787. DOI 10.1002/jcb.25476.

Li C, Dong Z, Zhang B, Huang Q, Liu G, Fu X (2020). Structural characterization and immune enhancement activity of a novel polysaccharide from Moringa oleifera leaves. Carbohydrate Polymers 234: 115897. DOI 10.1016/j. carbpol.2020.115897.

Li J, Chan MC, Yu Y, Bei Y, Chen P, Zhou Q, Cheng L, Chen L, Ziegler O, Rowe GC, Das S, Xiao J (2017). miR-29b contributes to multiple types of muscle atrophy. Nature Communications 8: 1704. DOI 10.1038/ncomms15201.

Li W, Dai RJ, Yu YH, Li L, Wu CM, Luan WW, Meng WW, Zhang XS, Deng YL (2007). Antihyperglycemic effect of Cephalotaxus sinensis leaves and GLUT-4 translocation facilitating activity of its flavonoid constituents. Biological and Pharmaceutical Bulletin 30: 1123-1129. DOI 10.1248/ bpb.30.1123.

Liu L, Li TM, Liu XR, Rai YP, Li J, Tang N, Wang XR (2019). MicroRNA-140 inhibits skeletal muscle glycolysis and atrophy in endotoxin-induced sepsis in mice via the WNT signaling pathway. American Journal of PhysiologyCell Physiology 317: C189-C199. DOI 10.1152/ ajpcell.00419.2018.
Mascaraque C, González R, Suárez MD, Zarzuelo A, de Medina FS, Martínez-Augustin O (2015). Intestinal anti-inflammatory activity of apigenin $\mathrm{K}$ in two rat colitis models induced by trinitrobenzenesulfonic acid and dextran sulphate sodium. British Journal of Nutrition 1134: 618-626. DOI 10.1017/ S0007114514004292.

Meng Q, Qi X, Fu Y, Chen Q, Cheng P, Yu X, Sun X, Wu J, Li W, Zhang Q, Li Y, Wang A, Bian H (2020). Flavonoids extracted from mulberry (Morus alba L.) leaf improve skeletal muscle mitochondrial function by activating AMPK in type 2 diabetes. Journal of Ethnopharmacology 248: 112326. DOI 10.1016/j.jep.2019.112326.

Miean KH, Mohamed S (2001). Flavonoid (myricetin, quercetin, kaempferol, luteolin, and apigenin) content of edible tropical plants. Journal of Agricultural and Food Chemistry 49: 3106-3112. DOI 10.1021/jf000892m.

Mulkey DK, Ritucci NA, Henderson RA, Putnam RW, Dean JB (2003). Reactive oxygen species (ROS) and chemical oxidants decrease intracellular $\mathrm{pH}\left(\mathrm{pH}_{\mathrm{i}}\right)$ of solitary complex neurons in rat brainstem slices. FASEB Journal 17: A15.

Nabavi SF, Khan H, D'onofrio G, Šamec D, Shirooie S, Dehpour AR, Argüelles S, Habtemarian S, Sobarzo-Sanchez E (2018). Apigenin as neuroprotective agent: Of mice and men. Pharmacological Research 128: 359-365. DOI 10.1016/j. phrs.2017.10.008.

Narasimhan M, Rajasekaran NS (2016). Exercise, Nrf2 and antioxidant signaling in cardiac aging. Frontiers in Physiology 7: 11488. DOI 10.3389/fphys.2016.00241.

Ogura Y, Kitada M, Xu J, Monno I, Koya D (2020). CD38 inhibition by apigenin ameliorates mitochondrial oxidative stress through restoration of the intracellular $\mathrm{NAD}^{+} / \mathrm{NADH}$ ratio and Sirt3 activity in renal tubular cells in diabetic rats. Aging-US 12: 11325-11336. DOI 10.18632/aging.103410.

Pipis M, Rossor AM, Laura M, Reilly MM (2019). Next-generation sequencing in Charcot-Marie-Tooth disease: opportunities and challenges. Nature Reviews Neurology 15: 644-656. DOI 10.1038/s41582-019-0254-5.

Quan W, Ma SB, Zhu YR, Shao Q, Hou JX, Li XQ (2020). Apigenin-7-Obeta-d-(6"-p-coumaroyl)-glucopyranoside reduces myocardial ischaemia/reperfusion injury in an experimental model via regulating the inflammation response. Pharmaceutical Biology 58: 80-88. DOI 10.1080/13880209.2019.1701043.

Rienzo MD, Antonioli M, Fusco C, Liu Y, Mari M, Orhon I, Refolo G, Germani F, Corazzari M, Romagnoli A, Ciccosanti F, Mandriani B, Pellico MT, De La Torre R, Ding H, Dentice M, Neri M, Ferlini A, Reggiori F, Kulesz-Martin M, Piacentini M, Merla G, Fimia GM (2019). Autophagy induction in atrophic muscle cells requires ULK1 activation by TRIM32 through unanchored K63-linked polyubiquitin chains. Science Advances 5: eaau8857. DOI 10.1126/sciadv.aau8857.

Rosa-Caldwell ME, Greene NP (2019). Muscle metabolism and atrophy: let's talk about sex. Biology of Sex Differences 10: 67. DOI 10.1186/s13293-019-0257-3.

Rychahuo PG, Murillo CA, Evers BM (2005). Targeted RNA interference of PI3K pathway components sensitizes colon cancer cells to TNF-related apoptosis-inducing ligand (TRAIL). Surgery 138: 391-397. DOI 10.1016/j.surg.2005.05.012.

Shiota C, Abe T, Kawai N, Ohno A, Teshima-Kondo S, Mori H, Terao J, Tanaka E, Nikawa T (2015). Flavones inhibit LPSinduced atrogin-1/MAFbx expression in mouse $\mathrm{C} 2 \mathrm{C} 12$ skeletal myotubes. Journal of Nutritional Science and Vitaminology 61: 188-194. DOI 10.3177/jnsv.61.188. 
Sun X, Han F, Lu Q, Li X, Ren D, Zhang J, Han Y, Xiang YK, Li J (2020). Empagliflozin ameliorates obesity-related cardiac dysfunction by regulating sestrin2-mediated AMPK-mTOR signaling and redox homeostasis in high-fat diet-induced obese mice. Diabetes 69: 1292-P. DOI 10.2337/db20-1292-P.

Tan YQ, Li Q, Wang L, Chiu-Leung LC, Leung LK (2020). The livestock growth-promoter zeranol facilitates GLUT4 translocation in 3T3 L1 adipocytes. Chemosphere 253: 126772. DOI 10.1016/j.chemosphere.2020.126772.

Trinchese G, Cavaliere G, Cimmino F, Catapano A, Carta G, Pirozzi C, Murru E, Lama A, Meli R, Bergamo P, Banni S, Mollica MP (2020). Decreased metabolic flexibility in skeletal muscle of rat fed with a high-fat diet is recovered by individual CLA isomer supplementation via converging protective mechanisms. Cells 9: 823. DOI 10.3390/cells9040823.

Verhavert Y, De Martelaer K, Van Hoof E, van der Linden E, Zinzen $\mathrm{E}$, Deliens $\mathrm{T}$ (2020). The association between energy balancerelated behavior and burn-out in adults: a systematic review. Nutrients 12: 397. DOI 10.3390/nu12020397.

Wang DT, Yang YJ, Zou XH, Zhang J, Zheng ZN, Wang ZW (2020). Antioxidant apigenin relieves age-related muscle atrophy by inhibiting oxidative stress and hyperactive mitophagy and apoptosis in skeletal muscle of mice. The Journals of Gerontology: Series A 75: 2081-2088. DOI 10.1093/gerona/glaa214.

Wang J, Liu Y, Xiao L, Zhu L, Wang Q, Yan T (2014). Antiinflammatory effects of apigenin in lipopolysaccharideinduced inflammatory in acute lung injury by suppressing COX-2 and NF-кB pathway. Inflammation 37: 2085-2090. DOI 10.1007/s10753-014-9942-X.

Xu X, Li M, Chen W, Yu H, Yang Y, Hang L (2016). Apigenin attenuates oxidative injury in ARPE-19 cells thorough activation of $\mathrm{Nrf} 2$ pathway. Oxidative Medicine and Cellular Longevity 2016: 1-9. DOI 10.1155/2016/4378461 .

Yang RY, Lin S, Kou G (2008). Content and distribution of flavonoids among 91 edible plant species. Asia Pacific Journal of Clinical Nutrition 17: 275-279.
Yang X, Yang JL, Hu J, Li XQ, Zhang XJ, Li ZL (2015). Apigenin attenuates myocardial ischemia/reperfusion injury via the inactivation of p38 mitogen-activated protein kinase. Molecular Medicine Reports 12: 6873-6878. DOI 10.3892/ mmr.2015.4293.

Yatsenko AS, Kucherenko MM, Xie Y, Aweida D, Urlaub H, Scheibe RJ, Cohen S, Shcherbata HR (2020). Profiling of the musclespecific dystroglycan interactome reveals the role of Hippo signaling in muscular dystrophy and age-dependent muscle atrophy. BMC Medicine 18: 3. DOI 10.1186/s12916-0191478-3.

Yin Y, Lu L, Wang D, Shi Y, Wang M, Huang Y, Chen D, Deng C, Chen J, Lv P, Wang Y, Li C, Wei L (2015). Astragalus polysaccharide inhibits autophagy and apoptosis from peroxide-induced injury in $\mathrm{C} 2 \mathrm{C} 12$ myoblasts. Cell Biochemistry and Biophysics 73: 433-439. DOI 10.1007/ s12013-015-0659-8.

Zhang S, Xu S, Duan H, Zhu Z, Yang Z, Cao J, Zhao Y, Huang Z, Wu Q, Duan J (2019). A novel, highly-water-soluble apigenin derivative provides neuroprotection following ischemia in male rats by regulating the ERK/Nrf2/HO-1 pathway. European Journal of Pharmacology 855: 208-215. DOI 10.1016/j.ejphar.2019.03.024.

Zhang TZ, Yan TH, Juan D, Wang SM, Yang HL (2015). Apigenin attenuates heart injury in lipopolysaccharide-induced endotoxemic model by suppressing sphingosine kinase $1 /$ sphingosine 1-phosphate signaling pathway. ChemicoBiological Interactions 233: 46-55. DOI $10.1016 /$ j. cbi.2014.12.021.

Zhang X, Hong YL, Xu DS, Feng Y, Zhao LJ, Ruan KF, Yang XJ (2014). A review of experimental research on herbal compounds in amyotrophic lateral sclerosis. Phytotherapy Research 28: 9-21. DOI 10.1002/ptr.4960.

Zhou S, Jiang J (2019). Anti-fatigue effects of active ingredients from traditional Chinese medicine: A review. Current Medicinal Chemistry 26: 1833-1848. DOI 10.2174/ 0929867324666170414164607. 